Revue d'histoire de l'Amérique française

REVUE D.HISTOIRE DE L'AMÉRIQUE FRANÇAISE

\title{
Sous les feux croisés de l'histoire et de l'anthropologie : la famille en Europe
}

\section{Martine Segalen}

Volume 39, numéro 2, automne 1985

Histoire de la famille

URI : https://id.erudit.org/iderudit/304348ar

DOI : https://doi.org/10.7202/304348ar

Aller au sommaire du numéro

Éditeur(s)

Institut d'histoire de l'Amérique française

ISSN

0035-2357 (imprimé)

1492-1383 (numérique)

Découvrir la revue

Citer cet article

Segalen, M. (1985). Sous les feux croisés de l'histoire et de l'anthropologie : la famille en Europe. Revue d'histoire de l'Amérique française, 39(2), 163-184.

https://doi.org/10.7202/304348ar d'utilisation que vous pouvez consulter en ligne.

https://apropos.erudit.org/fr/usagers/politique-dutilisation/ 


\title{
SOUS LES FEUX CROISÉS DE L'HISTOIRE ET DE L'ANTHROPOLOGIE: LA FAMILLE EN EUROPE
}

\author{
MARTINE SEGALEN \\ Centre d' ethnologie française \\ Musée national des arts et traditions populaires
}

Proposer un bilan de la production récente de l'histoire de la famille en Europe et notamment en France et en Angleterre - la question ne paraît simple qu'au premier coup d'oeil car elle recoupe de fait plusieurs interrogations: celle de la genèse (quand faire commencer ce bilan), celle de l'internationalisation du champ (études conduites par des Français sur la France et des Anglais sur l'Angleterre, par des Anglais sur la France et vice versa), celle enfin des limites d'une discipline qui se découpe en sous-champs sécants (démographie historique, histoire des mentalités) et aux frontières d'autres disciplines comme l'anthropologie et la sociologie.

Une constatation préalable: la masse des travaux, le volume des publications consacrées à la famille dans la multitude de ses facettes est aujourd'hui considérable. La question posée, difficile, vient donc à point, mais elle impose un classement a posteriori que le temps seul rend possible, et qui n'engage bien entendu que le chercheur qui en prend la responsabilité. D'autres se sont récemment livrés à ce périlleux exercice tels Gérard Soliday ${ }^{1}$, ou encore Lawrence Stone ${ }^{2}$ qui recensent pour différentes thématiques les principales positions problématiques. Un autre parti a été pris ici qui tente de concilier l'inconciliable: le thématique et le chronologique; de ce fait, certains des travaux et mouvements de pensée présentés sous telle rubrique pourraient sans doute figurer ailleurs.

La recherche sur la famille est née de la conjonction d'une double interrogation, celle née d'une discipline, la démographie historique, et celle formulée hors du champ scientifique à proprement parler. Aux lendemains de la seconde guerre mondiale, les experts s'interrogent sur le «baby boom» français, en opposition avec le mouvement des naissances que connaissait la France dans les années d'avant-guerre; les démographes souhaitent alors replacer ce mouvement dans la longue durée de l'histoire des populations: une technique remarquable, celle

\footnotetext{
1 Gérard L. Soliday, History of Family and Kinship: A Select International Bibliography (New York, 1980).

Lawrence Stone, «Family History in the 1980's. Past Achievements and Future Trends», Journal of Interdisciplinary History, 12 (1981): 51-87.
} 
de la reconstitution des familles va les y aider, et les conduire à un ensemble d'interrogations sur le couple, la fécondité, la place de l'enfant. On peut ainsi déceler un premier mouvement qui conduit de l'histoire des populations à celle des mentalités. Les travaux commencent alors à s'accumuler qui viennent rencontrer de plein fouet les mouvements d'idées associés aux années 1968, et aux mouvements féministes. Se développe le thème de la «crise» de la famille. Paradoxalement en ces années, la famille se portait tout à fait bien, si l'on veut en juger par les statistiques qui l'enserrent: jamais on ne s'était tant marié, jamais on ne s'était marié si jeune, jamais on n'avait fait tant d'enfants, jamais on n'avait si peu divorcé. Aujourd'hui alors que toutes ces statistiques se sont renversées, on pourrait parler de crise de la famille, et paradoxalement, le thème ne fait plus recette!

Quoi qu'il en soit de cette ironie du sort, les années 1968 ont interpellé les historiens qui, scientifiques dans leur siècle, voulaient pouvoir référer la situation contemporaine qu'ils vivaient à celle que connaissaient les périodes de leur spécialité. Ainsi voit-on se développer un intérêt pour la famille à l'époque moderne, comme au Moyen Age, à l'époque romaine ou barbare, périodes qui sont hors du champ de la démographie historique. Les historiens recherchent les documents les plus aptes à faire pénétrer dans la réalité la plus quotidienne et la plus intime. L'histoire de la famille est donc exemplaire de ce champ de la nouvelle histoire développé notamment par l'école des Annales.

Il serait toutefois erroné de penser que la recherche sur la famille s'est développée en France en un champ nationalement clos. Qu'il s'agisse de la démographie, de l'histoire, de l'anthropologie et dans une moindre mesure de la sociologie, le dialogue international a été une donnée constitutive marquante du champ. Conférences, échanges, publications (même si dans ce domaine, les anglophones traduisent beaucoup plus volontiers que ne le font les francophones: il faudrait entrer ici dans l'étude de la structure universitaire de l'édition en France et en Angleterre, ce qui est hors de notre propos) ont permis un dialogue constant entre Français et Anglais, et aussi Américains et Canadiens. Cette coopération a permis non seulement des comparaisons internationales, mais aussi un échange de concepts et de méthodes qui ont mutuellement fécondé les diverses recherches. L'internationalisation du champ, le va-et-vient d'idées entre les pays d'Europe et d'Amérique du Nord est particulièrement frappant dans le champ de l'anthropologie sociale; qu'il s'agisse de la parenté, du groupe domestique, des règles de mariage, du mode de transmission des biens, on observe un dialogue extrêmement riche entre anthropologues et historiens d'une part, entre école anthropologique anglaise et française d'autre part.

Si l'on examine le bilan des quinze années écoulées on peut dire que la connaissance théorique sur la famille a progressé: l'évolution- 
nisme qui caractérisait la vision du champ familial n'est plus de mise et l'on admet la variabilité des structures familiales. Au flou et à la polysémie du terme «famille», on a substitué la rigueur des concepts analytiques de la parenté, capables d'éclairer les interrelations entre changement social et changement familial.

\section{Genèse d'un objet: la famille au croisement de la démographie historique et de l'histoire des mentalités}

Quatre parrains (pas de marraines) se sont penchés sur le berceau du bébé «histoire de la famille en Europe», deux parrains anglais et deux parrains français qui se répondent en couples dans leur approche et leur influence sur le monde scientifique. D'un côté, Philippe Ariès et Peter Laslett, de l'autre John Hajnal et Louis Henry. Les deux premiers ont lancé des livres aux thèmes nouveaux, appuyés autant sur des intuitions que sur des recherches, et qui n'ont cessé d'inspirer au cours des vingt années qui suivirent les travaux des scientifiques du sérail. Il s'agit de l'Histoire des populations françaises et de leurs attitudes devant la vie depuis le XVIIIe siècle ${ }^{3}$, et de The World We Have Lost ${ }^{4}$, qui chacun à leur façon s'interrogeaient sur les problèmes de la vie privée, de l'âge au mariage, de la contraception et de la fécondité.

Par ailleurs l'article de John Hajnal, «European Marriage Patterns in Perspective» ${ }^{5}$, proposait une vision démographique tout à fait nouvelle du mariage, en comparant sociétés européennes et non-européennes: les premières étant caractérisées par un âge élevé au mariage, un faible écart d'âge entre les époux, contrairement aux sociétés généralement étudiées par les anthropologues où les jeunes mariées sortent de la puberté, et ont souvent un écart d'âge important avec leur époux. Ce modèle de mariage européen avait toutes sortes de conséquences sur les structures et les relations familiales. La méthode mise au point par Louis Henry et ses collègues de l'Institut national démographique permit d'apporter des confirmations massives à ces hypothèses très nouvelles (puisque les idées courantes étaient que l'on se mariait «autrefois» à l'âge de Roméo et Juliette). Pierre Goubert, dans sa thèse sur le Beauvaisis, avait montré la richesse des registres paroissiaux ${ }^{6}$. Louis Henry met au point une méthode systématique pour s'assurer de la qualité de leur exploitation. Un premier puis un «nouveau» manuel sont publiés en 1956 puis en 1965; entre-temps le célèbre Crulai était paru ${ }^{7}$,

\footnotetext{
3 Philippe Ariès, Histoire des populations françaises et de leurs attitudes devant la vie depuis le XVIIle siècle (Paris, Seuil, 1976). Première édition 1948.

4 Peter Laslett, The World We Have Lost (Londres, Methuen, 1965).

5 John Hajnal, «European Marriage Patterns in Perspective», Population in History, (1965): 101-147. D. V. Glass and D. E. C. Eversley, eds.

6 Pierre Goubert, Beauvais et le Beauvaisis de 1600 à 1730. Contribution à l'histoire sociale de la France du XVIIe siècle (Paris, Sevpen, 1960).

7 Étienne Gautier et Louis Henry, La population de Crulai, paroisse normande. Étude historique (Paris, Presses universitaires de France, 1958).
} 
modèle des modèles de ces monographies paroissiales qui apportaient des statistiques fiables sur la fécondité, la nuptialité, la mortalité, thèmes démographiques qui renvoient comme un miroir à des faits familiaux: le nombre des enfants et les espaces inter-génésiques, l'âge au mariage, le veuvage et le remariage, etc... Par ailleurs, le dialogue franco-anglais entre historiens démographes s'institutionnalise avec la création en 1964 du Cambridge Group for the History of Population and Social Structure.

Laissons de côté les résultats proprement démographiques de tous ces travaux qui sont analysés de façon critique par Jacques Dupâquier ${ }^{8}$ - et notamment l'existence de l'ancien régime démographique - pour nous intéresser aux prolongements relatifs à l'histoire de la famille à proprement parler. Implicitement, la famille (ou une certaine partie de la famille) est au coeur de la reconstitution entreprise dans la fiche Henry. Outre ses aspects purement démographiques, celle-ci décrit un ensemble de phénomènes sociaux, comme la fréquence du remariage et l'intervalle entre veuvage et remariage; elle analyse la fréquence des naissances, des morts d'enfants en bas âge, les intervalles inter-génésiques. Elle chiffre des interrogations, propose des évolutions. La fiche Henry cerne donc un ensemble de structures et de comportements qui relèvent d'une histoire sociale de la famille. Elle interpelle sur la sexualité, l'attitude à l'égard du corps (comme l'avait noté très tôt Philippe Ariès), la moralité et la mentalité, les relations entre parents et enfants, le mode d'élevage et d'éducation des enfants. Les réponses à ces questions sont contenues dans des documents non démographiques que les historiens avec une ingéniosité remarquable vont débusquer pour tenter d'analyser les courbes mises à jour par les démographes: actes notariés, archives judiciaires, textes des théologiens, livres de raison, observations conduites localement par des notables, récits des voyageurs, des folkloristes ou des préfets, etc...

Certains thèmes ont particulièrement fait florès. Ainsi la faiblesse des taux des naissances illégitimes, par exemple, a suscité de vastes débats sur la prétendue chasteté de nos ancêtres, entre tenants de l'ascèse sexuelle comme Pierre Chaunu ${ }^{9}$ et André Burguière ${ }^{10}$, et tenants d'une sexualité qui n'était pas seulement sublimée. Parmi ces derniers Jean-Louis Flandrin analysa la morale imposée par la puissance spirituelle dominante, en s'appuyant sur un ensemble de sources très neuves ${ }^{11}$. En Angleterre, l'observation des relations sexuelles hors

\footnotetext{
8 Jacques Dupâquier, Pour la démographie historique (Paris, Presses universitaires de France, 1984), $188 \mathrm{p}$.

9 Pierre Chaunu, La civilisation de l'Europe classique (Paris, Arthaud, 1966).

10 André Burguière, «De Malthus à Max Weber: le mariage tardif et l'esprit d'entreprise», Annales ESC, 27,4-5 (sept.-oct. 1972): 1118-1138.

11 Jean-Louis Flandrin, «Contraception, mariage et relations amoureuses dans l'Occident chrétien», Annales ESC, 24 (1969): 1370-1390; L'Eglise et le contrôle des naissances (Paris, Flammarion, 1970); Les amours paysannes (XVIe-XIXe siècles) (Paris, Gallimard-Julliard, 1975); «Repression and Change in the Sexual Life of Young People in Medieval and Early Modern Times", Journal of Family History, 2 (1977): 196-210; Un temps pour embrasser. Aux origines de la morale sexuelle occidentale (VIe-XIe siècles) (Paris, Le Seuil, 1983).
} 
mariage resta plus étroitement associée aux travaux statistiques de démographie historique, ce qui empêchait de répondre à l'intéressante hypothèse de recherche, à savoir l'existence d'une sous-culture, en quelque sorte, héréditaire de la bâtardise ${ }^{12}$.

La démographie historique avait également établi très fermement la forte fécondité et la forte mortalité, notamment des enfants en bas âge; cette constatation conduisait à s'interroger sur les causes de ces décès: les mères soignaient-elles leurs enfants? Tenaient-elles tant à la vie de ces nourrissons dont les naissances répétées devaient être un lourd fardeau? Philippe Ariès, une fois encore, avait ouvert le chemin en proposant des thèses hardies sur la découverte de l'enfance ${ }^{13}$, appuyées davantage sur des intuitions que sur des statistiques démographiques, et qui eurent d'ailleurs un retentissement considérable dans le monde anglo-saxon. Aucun autre ouvrage, selon Lawrence Stone, n'a autant stimulé la recherche en histoire de la famille.

En dépit de démonstrations parfois peu fiables, et de son imprécision irritante tant au sujet de la chronologie, de l'espace et de la classe sociale lorsque des exemples sont cités, il présentait cependant une nouvelle vision de l'expérience humaine passée et posait d'emblée la question majeure du moment et de la nature du changement familial d'une époque traditionnelle à l'époque contemporaine. ${ }^{14}$

La place de l'enfant dans la famille, les soins maternels étudiés à travers le prisme des traditions populaires furent l'objet de débats entre historiens et anthropologues. Des articles publiés dans un numéro spécial des Annales de démographie historique en 1973, à l'ouvrage de Françoise Loux ${ }^{15}$, aux travaux d'Yvonne Knibiehler et Catherine Fouquet ${ }^{16}$, les débats furent ardents entre ceux qui tenaient à la thèse de la négligence maternelle, voire de l'indifférence et ceux qui au contraire soutenaient que ces multiples recettes folkloriques étaient un moyen de pallier l'angoisse maternelle devant la fragilité de l'enfant ${ }^{17}$.

En Angleterre, les travaux sur l'enfance ont peut-être été moins nombreux. Citons le numéro spécial du Journal of Interdisciplinary History qui avait pour thème «The Family in History» paru à l'automne 1971. Ici l'histoire de la famille débouche sur celle de la médecine

\footnotetext{
12 Peter Laslett, «Long Term Trends in Bastardy in England», Family Life and Illicit Love in Earlier Generations (Cambridge, Cambridge University Press, 1977), 102-159.

13 Philippe Ariès, L'enfant et la vie familiale (Paris, Seuil, 1960).

14 Lawrence Stone, loc. cit., 72.

15 Françoise Loux, Le jeune enfant et son corps dans la médecine traditionnelle (Paris, Flammarion, 1978), $276 \mathrm{p}$.

16 Yvonne Knibiehler et Catherine Fouquet, L'histoire des mères du Moyen Age à nos jours (Paris, Montalba, 1980), $365 \mathrm{p}$.

17 Jacques Gelis, L'arbre et le fruit (Paris, Fayard, 1984), 611 p.
} 
populaire et sur celle du corps avec laquelle elle est bien souvent sécante. En Allemagne, l'histoire des mentalités et de la vie quotidienne reste encore étroitement liée aux développements de la démographie historique. Arthur Imhof notamment conduit des recherches dont certaines se centrent sur les conséquences sociales de l'allongement de l'espérance de vie ${ }^{18}$.

De la démographie historique à l'histoire des mentalités, plusieurs travaux ont analysé la famille comme un ensemble de pratiques avec leur logique indigène: citons par exemple ceux d'Yves Castan sur les codes de relations familiales ${ }^{19}$; ceux d'André Burgière et de Martine Segalen ${ }^{20}$ sur les rituels symboliques et sociaux qui scandent les âges de la vie et guident la constitution des couples; ceux qui la voient comme un ensemble de sentiments et d'attitudes au coeur desquelles l'analyse des livres de raison permet de pénétrer, en suivant la voie ouverte par Alan Macfarlane ${ }^{21}$. Ces quelques exemples attestent déjà de la rencontre entre historiens et anthropologues spécialistes des sociétés paysannes; rencontre quasiment fortuite. Sur d'autres thèmes de la famille elle fut plus systématique et mieux conceptualisée.

L'accumulation de ces travaux obligea à une remise en perspective de la vision qui avait jusqu'alors prévalu parmi les sociologues, celle d'un évolutionnisme qui voyait le passage inéluctable d'une «grande famille ancienne» à la «famille moderne nucléaire». Au 19e siècle Frédéric Le Play voyait dans cet état de fait le signe de la décadence des moeurs familiales, Talcott Parsons, au 20e siècle, la loi sociologique de l'industrialisation et de l'urbanisation.

Aujourd'hui les changements familiaux - qu'il s'agisse des structures, des sentiments ou des rapports entre société et famille - n'apparaissent plus comme linéaires ni unidimensionnels; malgré la variabilité des formes dans le temps et l'espace qui semble s'accentuer au fur et à mesure que se publient de nouveaux travaux, des typologies semblent se dessiner lorsqu'on associe aux structures des facteurs socio-économiques.

\footnotetext{
${ }_{18}$ Arthur E. Imhof, Die gewonnenen Jahre. Von den zunahme unserer lebensspanne seit dreihundert Jahren (München, Beck, 1981); Die verlorenen welten. Alltagsbewaltingung durch unsere vorfahren und weshalb wir uns heute so schwer damit tun (München, Beck, 1984). 1974).

Yves Castan, Honnêteté et relations sociales en Languedoc, 1715-1780 (Paris, Plon,

André Burguière, «Le rituel du mariage en France. Pratiques ecclésiastiques et pratiques populaires (XVIe-XVIIIe siècle», Annales ESC, 33,3 (mai-juin 1978): 637-649. Martine Segalen et Jocelyne Chamarat, Amours et mariages de l'ancienne France (Paris, Berger-Levrault, 1981), $180 \mathrm{p}$.

Alan Macfarlane, The Family Life of Ralph Josselin. A Seventeenth Century Clergyman (Cambridge, Cambridge University Press, 1970), 241 p.
} 


\section{L'abandon d'une vision évolutionniste de la famille}

La naissance d'une nouvelle vision de la famille est attachée à la découverte du groupe domestique ancien dont il devenait possible de mesurer la taille et la structure.

C'est moins par adoption systématique d'un concept, par ailleurs très travaillé par l'école anthropologique anglaise, avec les travaux de Meyer Fortes et Jack Goody, que par extraction quelque peu fortuite à partir des sources à leur disposition que les historiens anglais ont rencontré le groupe domestique ou ménage, «Household». De même que la fiche de famille s'imposait aux chercheurs français à partir des registres paroissiaux d'état civil ancien, de même le ménage a-t-il surgi des listes nominatives de recensement, documents beaucoup plus fréquents en Angleterre que dans la France ancienne, et capables, pensait-on initialement, de pallier les déficiences de l'état civil anglais rendant les reconstitutions de fiches de famille très difficiles puisque les noms de parents n'y figuraient pas, non plus que le nom de jeune fille des mères ${ }^{22}$. Toute personne qui travaille sur l'histoire de la famille connait la suite de cette histoire-là; le colloque international de Cambridge en 1969, le fameux Household and Family in Past Time ${ }^{23}$, qui proposait à la fois un cadre typologique d'analyse des recensements, et déjà une masse de résultats internationaux, avec des comparaisons quelquefois hasardeuses. Peter Laslett soutient dans l'introduction de son ouvrage que les groupes domestiques dans l'Angleterre (et l'Europe) pré-industrielle sont déjà de taille réduite et de structure simple: parents-enfants. La société pré-industrielle ne connait pas la «grande famille», et l'industrialisation n'a donc pas nucléarisé la famille, puisque la famille était nucléaire depuis longtemps. Et il va même jusqu'à retourner la proposition classique de la sociologie américaine des années 50: la famille n'a pas été nucléarisée par l'industrialisation, mais au contraire, c'est parce que la famille était réduite que l'industrialisation a pu décoller dans la société anglaise d'abord, et les sociétés nord-européennes ensuite.

Cette thèse eut un triple retentissement. D'abord, la critique de la typologie Laslett, et notamment de son comparatisme trop systématique, ont amené la production d'une masse considérable de travaux tant en Europe qu'en Amérique du Nord. En second lieu ses propositions jetaient un pont au-dessus des eaux du large fleuve séparant les rives de l'anthropologie, la démographie et l'histoire d'une part, et de la sociologie d'autre part. Même si les travaux présentés dans l'ouvrage s'appuyaient avant tout sur des données du $18 \mathrm{e}$ et du $19 \mathrm{e}$ siècles, la

\footnotetext{
22 E. A. Wrigley, ed., An Introduction to English Historical Demography (London, Weidenfeld and Nicolson, 1966).

${ }_{23}$ Peter Laslett et Richard Wall, eds., Household and Family in Past Time (Cambridge, Cambridge University Press, 1972), 623 p.
} 
visée théorique englobait les rapports entre changement social et changement familial jusqu'à l'époque contemporaine: saluons l'ampleur de la perspective.

Enfin, la famille apparaissait comme une instance agissante, sinon dynamique, capable de résister aux changements sociaux, de s'y adapter: la flexibilité de sa structure étant en quelque sorte garante de sa survie.

Les thèses initiales de Peter Laslett sur la quasi-universalité de la structure nucléaire de la famille ont fait place maintenant à une géographie et une chronologie plus élaborées, dans la mesure où se sont développées les analyses sur les modes de dévolution des biens, qui semblent souvent présenter des affinités structurales avec les types de groupes domestiques.

La famille paysanne de l'Europe pré-industrielle est associée à un système complexe de droits de propriété. Le mode de transmission de ces droits diffère d'une région à l'autre, tant dans sa règle que dans sa pratique, selon une combinatoire subtile qui associe ou dissocie l'héritage égalitaire/inégalitaire au mode de succession unique ou joint. L'étude de la reproduction des groupes domestiques se trouvait donc nécessairement associée à celle du mode de dévolution des biens ${ }^{24}$.

Ici encore anthropologues et historiens anglais apparaissent comme les pionniers dans ce champ d'analyse avec l'ouvrage dirigé par Jack Goody et al. ${ }^{25}$, s'appuyant d'ailleurs sur des articles antérieurs traitant de ces problèmes, notamment une synthèse publiée par Emmanuel Le Roy Ladurie ${ }^{26}$, et un cas finement analysé par Alain Collomp pour la France du sud-est. Dans la même veine, Lutz Berkner publiait une étude comparative de plusieurs sociétés de Basse-Saxe mettant en rapport régime démographique et mode de dévolution des biens ${ }^{27}$.

La géographie de la France semble se caractériser par trois types de modèles familiaux: un Sud et un Centre qui associent à la famillesouche un régime de dévolution des biens qui fait un héritier unique ${ }^{28}$, l'Ouest et la Bourgogne qui seraient des espaces égalitaires associés à

\footnotetext{
24 Georges Augustins, «Division égalitaire des patrimoines et institutions de l'héritier», Archives européennes de sociologie, 20 (1979): 127-141; «Esquisse d'une comparaison des systèmes de perpétuation des groupes domestiques dans les sociétés paysannes européennes», Archives européennes de Sociologie, 23 (1982): 39-69.

${ }_{25}$ Jack Goody, Joan Thirsk, E. P. Thompson, eds., Family and Inheritance (Cambridge, Cambridge University Press, 1976), $421 \mathrm{p}$.

26 Emmanuel Le Roy Ladurie, «Système de la coutume. Structures familiales et coutumes d'héritage en France au XVIe siècle», Annales ESC, 27,4-5 (juil.-oct. 1972): 825-846.

27 Lutz Berkner, «Peasant Households Organization and Demographic Change in Lower Saxony», in Population Patterns in the Past (New York, Academic Press, 1977), 53-71. (Ronald Demos Lee, ed.)

${ }_{28}$ Alain Collomp, La maison du père (Paris, Presses universitaires de France, 1983), 342 p.
} 
une famille nucléaire ${ }^{29}$, tandis que certaines poches, comme la FrancheComté associeraient un type de famille communautaire (frérèche) à un régime égalitaire, en réalité fortement favorable aux garçons ${ }^{30}$. L'analyse d'éco-types paysans dans diverses régions de Suède met en lumière des corrélations du même type ${ }^{31}$ : de même l'Europe du Centre et les pays baltes sont souvent caractérisés par des types de familles communautaires qui, exploitant en commun de vastes domaines, devaient s'appuyer sur la main-d'oeuvre de couples nucléaires qui regroupaient leurs forces $^{32}$.

Bien entendu, en suggérant l'existence de modèles contrastés de structure familiale en Europe, on n'ignore pas les changements que peuvent prendre ces structures au long du cycle de la vie familiale. Depuis les travaux de Jack Goody ${ }^{33}$, la critique classique de Lutz Berkner $^{34}$ et l'ouvrage dirigé par Jean Cuisenier ${ }^{35}$, l'analyse formelle des groupes domestiques s'applique à prendre en compte la dimension chronologique. Ainsi, chaque ménage, dans le cadre normatif de la famille-souche, nucléaire, ou complexe, connaît des phases d'expansion ou de contraction selon les aléas de la démographie, l'écoulement du temps, ou le niveau de richesse du groupe considéré.

Les hypothèses de Peter Laslett et du Cambridge Group, si elles ont renouvelé profondément la vision des sociétés paysannes, ont été intégrées également par les historiens s'intéressant au monde urbain et ouvrier, bien davantage en Angleterre qu'en France d'ailleurs. Michael Anderson $^{36}$ renversait lui aussi la vision relative aux transformations familiales, en montrant que, dans les cités cotonnières du Lancashire, le groupe domestique était beaucoup plus souvent complexe ou étendu que dans les villages dont étaient originaires les migrants. Alors que sur les modestes tenures paysannes, la famille était toujours nucléaire, dans les villes ouvrières, elle associait souvent trois générations: les conditions de salaire, de logement, ainsi que l'absence de toute aide

\footnotetext{
29 Marie-Claude Pingaud, Paysans en Bourgogne. Les gens de Minot (Paris, Flammarion, 1978), $300 \mathrm{p}$.

30 Michèle Salitot-Dion, «Évolution économique, cycle familial et transmission patrimoniale à Nussey», Études rurales, 68 (oct.-déc. 1977): 23-53; «Régime matrimonial et organisation familiale en Franche Comté», Ethnologie française, 4 (oct.-déc. 1978): 321-328.

${ }_{31}$ Orvar Lofgren, «Family and Household Among Scandinavian Peasants: an Exploratory Essay», Ethnologia Scandinavia (1974): 17-33.

32 Richard Wall, ed., avec Jean Robin et Peter Laslett, Family Forms in Historic Europe (Cambridge, Cambridge University Press, 1983), $606 \mathrm{p}$.

33 Jack Goody, ed., The Developmental Cycle in Domestic Groups (Cambridge, Cambridge University Press, 1971).

${ }_{34}$ Lutz Berkner, «The Stem Family and the Developmental Cycle of the Peasant Household: an XVIIIth Century Austrian Example», American Historical Review, 77 (1972): 400402 .

Jean Cuisenier, dir., assisté de Martine Segalen, Le cycle de la vie familiale dans les sociétés européennes (Paris, Mouton, 1977), $494 \mathrm{p}$.

36 Michael Anderson, Family Structure in Nineteenth Century Lancashire (Cambridge, Cambridge University Press, 1971), $230 \mathrm{p}$.
} 
sociale rendaient cette co-habitation indispensable, même si elle était subie difficilement.

Hans Medick ${ }^{37}$ fait des observations similaires à propos de la famille proto-industrielle, c'est-à-dire les foyers qui produisent à domicile des produits destinés à l'industrie, mais qui sont insérés dans la communauté villageoise dont ils partagent les valeurs et les coutumes. Le ménage est totalement identifié à l'unité économique de production, et comme dans les grands domaines qui nécessitent une nombreuse maind'oeuvre, il lui faut mettre au travail les aïeuls, les enfants, les collatéraux, compte tenu de la faiblesse des revenus tirés de l'activité industrielle. Là encore, le ménage a toutes les chances de se trouver «complexifié» du fait des conditions de la production. De plus, il possède quelques traits d'une famille «moderne». Dans la mesure où les deux époux sont maintenant salariés, les rôles traditionnels semblent se modifier vers une répartition plus égalitaire. La structure du groupe domestique et le travail féminin ont par ailleurs des interrelations: ainsi voit-on l'importance des pensionnaires dans les ménages où la femme n'a pas d'activité industrielle et compense en quelque sorte ce manque à gagner par l'entretien d'un étranger au foyer. Les groupes domestiques anglais et français semblent se distinguer sur ce point, mais il faudrait d'autres études pour approfondir cette différence, comme il faudrait évaluer la spécificité du phénomène des enfants placés en service qui a également une incidence sur la taille et la structure des groupes domestiques. Phénomène classique en Angleterre, plutôt rare en France où la condition de domestique est associée à la pauvreté, même si c'est une forme d'apprentissage.

Le rejet d'une vision évolutionniste, la recherche de corrélations socio-économiques et démographiques pour expliquer la variabilité des structures des ménages n'épuisent cependant pas le problème de l'articulation entre changements sociaux et changements familiaux. Il a souvent été reproché à Peter Laslett d'avoir réduit toute la famille au groupe domestique co-résident. De fait, il existe des relations entre groupes domestiques et individus apparentés, mais qui ne partagent pas le même espace résidentiel. Qu'en est-il de ces rapports? Un second ensemble de recherches semble pouvoir être organisé autour de ces questions.

\section{L'importance des usages sociaux de parenté}

Jack Goody soulignait à juste titre ${ }^{38}$ que la famille nucléaire était une unité significative dans toutes les sociétés, même celles qui s'organisent selon des systèmes de parenté élaborés en clans ou lignages,

\footnotetext{
37 Hans Medick, «The Proto-Industrial Family Economy: the Structural Function of Household and Family During the Transition From Peasant Society to Industrial Capitalism», Social History, 3 (1976): 291-315. 124.

Jack Goody, «The Evolution of the Family», in P. Laslett and R. Wall, op. cit., 103-
} 
et que, ce qui était nouveau, c'était moins la «nucléarisation» de la famille, que les changements dans les relations entre familles nucléaires apparentées, suggérant lui aussi un amenuisement de ces liens avec l'urbanisation et l'industrialisation.

Nombre de recherches d'ethno-histoire ont au contraire mis en lumière l'importance du rôle social de la parenté dans les sociétés paysannes, son imbrication avec le politique et l'économique. D'autre part le rôle actif des réseaux de parenté dans l'organisation des mouvements migratoires des campagnes vers les villes, dans la structuration des groupes nouvellement implantés en milieu urbain a été vérifié dans maints contextes sociaux ou ethniques ${ }^{39}$. Dans cette perspective, le fait familial cessait d'être identifié uniquement au groupe domestique et s'ouvrait au groupe d'apparentés plus large dont l'importance ne cesse d'être redécouverte, qu'on considère ses usages symboliques - le fonctionnement de la mémoire familiale par exemple - ou ses usages sociaux.

Il est un usage social de la parenté sur lequel les anthropologues de la France se sont tout particulièrement penchés, en cherchant à repérer, dans le cadre des théories de Claude Lévi-Strauss ${ }^{40}$, d'éventuelles régularités au sein des sociétés complexes. Dans ces dernières, la parenté, on le sait, ne constitue qu'un des éléments du système social, et le choix du conjoint, en dehors de quelques parents prohibés, est théoriquement libre. Françoise Zonabend, Tina Jolas, Yvonne Verdier ${ }^{41}$, à partir de l'étude monographique du village bourguignon de Minot, analysent la parenté vécue et les rituels des âges de la vie. De ces recherches date l'invention du concept de «renchaînement d'alliance» entre lignées, si souvent repris aujourd'hui. Par ailleurs, l'article de Pierre Bourdieu, «Célibat et condition paysanne» ${ }^{42}$, s'il resta immédiatement sans réponse, allait ensemencer le terreau de discussions futures, à partir d'une analyse subtile du mode de dévolution des biens et des stratégies matrimoniales en Béarn. Des historiens et d'autres anthropologues se retrouvaient sur le même terrain thématique à partir de la problématique plus démographique de l'endogamie, comme André Burguière $^{43}$, Jean-Marie Gouesse ${ }^{44}$, Martine Segalen ${ }^{45}$. Les rapports

39 Martine Segalen Sociologie de la Famille (Paris, A. Colin, 1981), 283 p.

40 Claude Lévi-Strauss, Les structures élémentaires de la parenté (Paris, Mouton, 1968),

$\mathrm{p}_{41} \quad$ Tina Jolas, Yvonne Verdier et Françoise Zonabend, «Parler famille», L'Homme, 10,3 (1970); Françoise Zonabend, «Le très proche et le pas trop loin», Ethnologie française, 11,4 (1981): 311-318; Tina Jolas et Françoise Zonabend, «Cousinage, voisinage», in Echanges et communications, mélanges offerts à Claude Lévi-Strauss (Paris, Mouton, 1970).

42 Pierre Bourdieu, «Célibat et condition paysanne», Études rurales, 5-6 (1962): 83-134.

43 André Burguière, «Endogamie et communauté villageoises: pratique matrimoniale à Romainville au XVIIIle siècle», Annales de démographie historique (1979): 313-336.

44 Jean-Marie Gouesse, «Parenté, famille et mariage en Normandie aux XVIIe et XVIIIe siècles", Annales ESC, 4-5 (juill.-oct. 1972): 1139-1154.

${ }_{45}$ Martine Segalen, Nuptialité et alliance, le choix du conjoint dans une commune de l'Eure (Paris, G. P. Maisonneuve et Larose, 1972), 142 p. 
entre système d'alliance et type de dévolution des biens sont à l'heure actuelle un des thèmes d'étude dont la discussion est la plus vive, stimulée par l'Exercice de la Parenté de Françoise Héritier ${ }^{46}$, consacré à la recherche d'une continuité entre sociétés élémentaires, semi-complexes et complexes.

Autour de ces thèmes se cristallise une riche production scientifique anthropologique et historique, dont les frontières sont d'autant plus floues que les anthropologues ont besoin de longues respirations chronologiques pour repérer d'éventuelles régularités matrimoniales. Pierre Lamaison ${ }^{47}$ a mis en évidence l'existence de lignées patrimoniales dans le Gévaudan, région préciputaire du Centre de la France; là, comme dans les régions pyrénéennes et celles du Sud-Ouest de la France, les lignées s'ancrent dans une propriété paysanne qui englobe les biens matériels et symboliques désignés par le terme de «maison», «oustal» en occitan. Le maintien intégral de la propriété familiale structure l'ensemble des stratégies, et l'auteur met en évidence des formes d'échanges restreints et d'échanges généralisés. Le type structurel d'union le mieux adapté au système d'héritage est le mariage simultané de l'aîné-héritier d'une maison avec une cadette-exclue d'une autre maison, tandis que le cadet de la première maison épouse l'héritière de l'autre. Les dots données par les maisons aux cadets sont de montant identique et de fait fictives: aucun argent ne sort qui viendrait affaiblir le patrimoine.

Une vision plutôt structuraliste ou structurelle tendrait à opposer à ce modèle celui qui règne dans les sociétés septentrionales et de l'Ouest de la France, caractérisées souvent par une grande mobilité des paysans qui ne sont pas propriétaires de leurs fermes. L'hypothèse a été faite que des lignées de successeurs joueraient le rôle des lignées de propriétaires ${ }^{48}$; ou encore que les mariages entre cousins seraient préférés pour remembrer à la troisième génération le patrimoine de l'ancêtre partagé à la seconde ${ }^{49}$. Une telle vision fonctionnaliste ne résiste pas à l'analyse. Dans les sociétés égalitaires, ce qui est préféré, c'est le renchaînement d'alliance au sein de la parentèle, ensemble de lignées dont la stabilité compense la mobilité individuelle des groupes domestiques. Ces figures matrimoniales unissent des conjoints appartenant à des lignées qui ont déjà contracté alliance, dans les générations antérieures, mais proches. Elles répondent à des finalités économiques ainsi on constate que les renchaînements les plus fréquents s'organisent au sein des parentèles paysannes les plus aisées -, mais aussi à des

\footnotetext{
Françoise Héritier, L'exercice de la parenté (Paris, EHESS, Gallimard/Le Seuil, 1981), $199 \mathrm{p}$

7 Pierre Lamaison et Élizabeth Claverie, L'impossible mariage. Violence et parenté en Gévaudan, 17e, 18e, 19e siècles (Paris, Hachette, 1982), $361 \mathrm{p}$

48 Idem.

49 Idem.
} 
finalités symboliques, comme le fait de rester entre soi, de constituer un réseau d'interconnaissance par lequel circulent les informations sur les fermes libres ou les terres à acquérir ${ }^{50}$.

L'opposition entre structure et stratégie n'est en fait pas pertinente si l'on prend en compte la dimension chronologique qui transforme les systèmes au cours du temps, et l'écart entre norme et pratique qui corrige souvent les effets supposés très tranchés entre systèmes de dévolution des biens. Dans les systèmes préciputaires, les pères essayaient parfois de ne pas trop défavoriser les autres héritiers ${ }^{51}$, tandis qu'en régime égalitaire, lorsqu'ils étaient propriétaires, les paysans s'efforçaient souvent de ne pas démembrer leurs exploitations.

Les recherches de Lamaison et Segalen s'appuient sur des reconstitutions de généalogies et des traitements informatiques. On peut en rapprocher le travail de Gérard Delille sur la famille et la propriété dans le Royaume de Naples du $15 \mathrm{e}$ au $19 \mathrm{e}$ siècle ${ }^{52}$ qui met également en évidence des régularités matrimoniales, à partir de livres de généalogies de familles nobles et bourgeoises.

On ne s'étonnera guère que les travaux anthropologiques anglais soient d'une inspiration sensiblement différente. Préparés dans la riche tradition des «community studies», les recherches anglaises sur ces thèmes ont été également pionnières, avec la célèbre étude de Arensberg et Kimball ${ }^{33}$ analysant le fonctionnement de la famille et de la communauté jusqu'à celle de Robin Fox ${ }^{54}$ qui montre chez les Tory Islanders l'existence d'un droit de propriété moins individuel qu'appartenant à un groupe de parents, avec une règle de résidence uxorilocale. Dans les deux cas, il s'agit de communautés d'origine celte marquées par un système de parenté spécifique. Les villages d'Angleterre sont moins souvent l'objet de ce type d'investigations, et l'étude conduite par Jean Robin sous la direction d'Audrey Richards sur Elmdon ${ }^{55}$ fait figure d'exception, tout comme l'analyse conjointe menée sur la parenté de ce même village par Marilyn Strathern ${ }^{56}$. Faut-il voir dans cette absence d'intérêt la justification de la thèse provocatrice d'Alan

\footnotetext{
50 Martine Segalen, Quinze générations de bas bretons. Parenté et société dans le pays Bigouden Sud 1720-1980 (Paris, Presses universitaires de France, 1985), 406 p.

51 Alain Collomp, "Alliance et filiation en Haute-Provence au XVIIle siècle», Annales ESC, 3 (mai-juin 1977): 445-477.

${ }_{52}$ Gérard Delille, Famille et propriété dans le Royaume de Naples XVe-XIXe siècles. Thèse de doctorat d'État, Université de Paris I, Faculté des Lettres et des Sciences humaines, 2 vol., 1981-1982, $953 \mathrm{p}$.

53 C. M. Arensberg et S. T. Kimball, Family and Community in Ireland (Cambridge, Harvard University Press, 1940).

54 Robin Fox, The Tory Islanders. A People of the Celtic Fringe (Cambridge, Cambridge University Press, 1978), $210 \mathrm{p}$.

55 Jean Robin, Elmdon, Continuity and Change in a North-West Essex Village 1861-1964 (Cambridge, Cambridge University Press, 1980), 260 p.

56 Marilyn Strathern, Kinship at the Core. An Anthropology of Elmdon, a Village in NorthWest Essex, in the Nineteenth-Sixties (Cambridge, Cambridge University Press, 1981), 301 p.
} 
Macfarlane ${ }^{57}$ selon laquelle la paysannerie n'existerait pas en Angleterre, car les supposées caractéristiques - et notamment caractéristiques familiales - en seraient absentes? Ce court ouvrage mérite une mention spéciale en raison des réactions nombreuses qu'il a stimulées. Comme c'est souvent le cas, c'est la vision que pose un anthropologue spécialiste de sociétés très différentes de la nôtre (c'est le cas d'Alan Macfarlane) qui renouvelle le plus vivement les cadres problématiques d'étude de notre société.

De même les concepts de l'analyse anthropologique de la parenté ont renouvelé certaines perspectives des médiévistes, comme Georges Duby et Jacques LeGoff ${ }^{88}$ dont les analyses sont évidemment déplacées en raison des sources depuis le peuple des paysans vers l'élite des chevaliers.

\section{Une histoire des femmes encore balbutiante}

Un des chapitres de l'histoire de la famille, l'histoire des femmes est en train de s'écrire en France, comme dans les autres pays d'Europe et d'Amérique du Nord. Là encore, les préoccupations contemporaines ont excité l'imagination des spécialistes du passé. Pour analyser la condition des femmes d'aujourd'hui, il faut pouvoir la référer à celle dont elles jouissaient ou pâtissaient autrefois. Ces études sur la femme sont le plus souvent sécantes avec celles de la famille, mais également la débordent ou l'ignorent en étudiant la femme dans tel ou tel contexte social, économique ou politique. D'un point de vue international, une différence marquante: la lenteur du développement des études féministes en France en regard de l'explosion des Women's Studies.

Michelle Perrot ${ }^{59}$ note qu'en France les études féministes se sont développées de façon éclatée dans diverses universités et lieux de recherche, qu'il n'y existe pas de cursus autonome d'études féminines, et pas de grande revue. Souvent ce sont des synthèses générales qui ont posé les questions les plus fondamentales à propos de la condition féminine. Les progrès économiques ont-ils profité autant à la femme qu'à la société en général? Dans sa Naissance de la famille moderne, Edward Shorter $^{60}$ se montre optimiste alors que James MacMillan ${ }^{61}$, verrait plutôt une stagnation de la condition féminine entre la fin du $19 \mathrm{e}$ et le début du $20 \mathrm{e}$ siècle. Pour dépasser des vues parfois trop idéologiques il faut

\footnotetext{
57 Alan Macfarlane, The Origins of English Individualism (Oxford, Basil Blackwell, 1978), $216 \mathrm{p}$.

Georges Duby, Le chevalier, la femme et le prêtre. Le mariage dans la France féodale (Paris, Hachette, 1981), 311 p. Georges Duby et Jacques LeGoff, Famille et parenté dans l'Occident médiéval (Rome, 1977).

59 Michelle Perrot, «Sur l'histoire des femmes en France», Revue du Nord, 63,250 (juilletseptembre 1981): 569-579.

60 Edward Shorter, Naissance de la famille moderne (Paris, Seuil, 1977), 379 p.

61 James MacMillan, Housewife of Harlot. The Place of Women in French Society (18701940) (Brighton, Harvester Press, 1981).
} 
savoir s'appuyer sur des analyses restituant les comportements dans un cadre social bien déterminé. Yvonne Verdier ${ }^{62}$, Martine Segalen ${ }^{63}$ se sont attachées à décrire les rôles symboliques et les pouvoirs de la femme dans la société paysanne. Louise Tilly et Joan Scott ${ }^{64}$ étudient les femmes ouvrières d'Anzin et de Roubaix et montrent le rôle de la femme comme gestionnaire des budgets familiaux, et comment celle-ci module son entrée sur le marché du travail en fonction du cycle de la vie familiale. La femme au travail, le travail des femmes sont l'objet de travaux nombreux; un numéro spécial du Mouvement social ${ }^{65}$ lui fut consacré; la femme à la maison et son travail domestique ont été réévalués de façon fort pertinente par Michelle Perrot ${ }^{66}$. Outre les paysannes et les ouvrières, les bourgeoises sont également l'objet d'investigations, soit à travers la famille, soit à travers le prisme de la domesticité qui l'entoure tant en France qu'en Angleterre ${ }^{67}$. Un ensemble de pistes de recherches est offert dans l'ouvrage dirigé par Jean-Paul Aron ${ }^{68}$; l'étude de la prostitution par Alain Corbin ${ }^{69}$ éclaire également les comportements familiaux de la bourgeoisie et suivent la montée de la «familialisation» du ménage ouvrier.

«Le regard éloigné» est ici nécessaire et ce sont les Anglo-Saxons qui proposent des pistes ou des synthèses relatives à la France. A propos de la femme bourgeoise, l'ouvrage de Théodore Zeldin ${ }^{70}$, celui de Bonnie Smith ${ }^{71}$ ont eu autant d'impact sur la recherche en France que celui de Louise Tilly et Joan Scott pour la famille ouvrière. Des synthèses provisoires sont par ailleurs proposées par la revue Pénélope qui centre chaque numéro sur un thème particulier: l'éducation, la presse, le bureau, les associations, la violence, la science, etc... La femme seule est l'objet d'un ouvrage dirigé par Arlette Farge et Christiane Klapisch-Zuber ${ }^{72}$; le corps de la femme est l'objet de l'inépuisable attention de chercheurs souvent masculins. Le dernier livre d'Edward Shorter ${ }^{73}$ suggère que la

\footnotetext{
62 Yvonne Verdier, Façons de dire, façons de faire. La laveuse, la couturière, la cuisinière (Paris, Gallimard, 1979), 373 p. $211 \mathrm{p}$. 1978). Louise Tilly et Joan Scott, Women, Work and Family (Holt, Rinehart and Winston,

65 Sous la direction de Michelle Perrot, «Travaux de femmes dans la France du XIXe siècle», Le Mouvement social (octobre-décembre 1978), 206 p.

66 Michelle Perrot, loc. cit., note 22.

67 Léonore Davidoff, The Best Circles: Women and Society in Victorian England (Londres, 1973). 1980), $248 \mathrm{p}$

69 Alain Corbin, Les filles de noce (Paris, Aubier-Montaigne, 1978), $571 \mathrm{p}$

70 Théodore Zeldin, Histoire des passions françaises. Ambition et Amour (Paris, Encres, 1978), 421 p. Tome 1.

71 Bonnie G. Smith, Ladies of the Leisure Class. The Bourgeoises of Northern France in the Nineteenth Century (Princeton, Princeton University Press, 1981), 304 p.

72 Arlette Farge et Christiane Klapisch-Zuber, dir., Madame ou Mademoiselle. Itinéraires de la solitude féminine XVIIIe-XXe siècles (Paris, Montalba, 1984), 302 p.

${ }^{73}$ Edward Shorter, History of Women's Bodies (New York, Basic Books, 1982), 398 p.
} 
pensée féministe n'a pu se constituer comme mouvement autonome et revendicatif que lorsque la médecine a délivré - tout récemment - le corps féminin des fatalités que la «nature» semblait y avoir attachées.

Le regard féministe, issu de l'anthropologie marxiste, s'est posé sur le travail domestique et son rôle économique dans la société marchande ${ }^{74}$, comme sur les catégories sociologiques utilisées pour examiner les professions, les classes, ou construire les index de mobilité sociale. Ces dernières sont évidemment établies d'un point de vue strictement masculin, sans inclure les charges spécifiques que les femmes connaissent du fait de leurs maternités ou de leur surcharge de travail si elles exercent une activité salariée. En dépit de son intérêt, une telle perspective, purement idéologique, se révèle inefficace car elle analyse les femmes de l'extérieur, sans tenir compte des stratégies vécues. Elle débouche souvent sur des analyses largement abstraites, détachées de la réalité et qui ne font guère progresser la connaissance sur la condition féminine et son histoire. Comme aux États-Unis et en Angleterre, mais certainement sur une moins large échelle, les travaux s'accumulent maintenant qui nous montrent que pas plus qu'il n'y a une famille, mais des variétés de types familiaux, pas plus n'y-a-t-il une femme: il conviendrait de parler «des» femmes, dans la variété des périodes et des contextes. Un bilan récent des recherches conduites sur les femmes en France ${ }^{75}$ parvient aux mêmes conclusions que le regard évaluatif porté depuis les États-Unis ${ }^{76}$. L'histoire des femmes est inséparable de celle des rapports entre les sexes, des différences entre les sexes. A trop s'interroger sur la nature du corps, des espaces, des représentations de la femme, on finit par l'enfermer dans le ghetto duquel on voulait la faire sortir.

\section{Du bilan aux perspectives}

Les premières synthèses suggérées par l'accumulation de données neuves sur la famille étaient empreintes d'un évolutionnisme. Si elles avaient l'immense intérêt de coudre ensemble le patchwork des recherches conduites à différentes époques et dans divers contextes socioculturels, leur simplicité, voire leur simplisme leur valurent souvent de vigoureuses critiques. Edward Shorter ${ }^{77}$ voyait l'émergence d'une famille moderne appuyée sur une vision ethnocentriste et unilinéaire des transformations familiales. Saluons au passage l'immense masse documentaire qu'il avait embrassée, même si l'usage de certaines sources

\footnotetext{
74 Andrée Michel, dir., Les femmes dans la société marchande (Paris, Presses universitaires de France, 1978), 256 p.

${ }_{75}$ Michelle Perrot, dir., Une histoire des femmes est-elle possible? (Paris, Rivages, 1984), $225 \mathrm{p}_{76}$

76 Joan W. Scott, «Survey Articles. Women in History. The Modern Period», Past and Present, 101 (1983): 141-157.

${ }_{77}$ Edward Shorter, Naissance de la famille moderne (Paris, Seuil, 1977), $379 \mathrm{p}$.
} 
était quelque peu naïve. Par ailleurs, il suggérait des hypothèses intéressantes sur la naissance d'un sentiment amoureux parmi les couches démunies de la société, libérées des contraintes patrimoniales. Lawrence Stone ${ }^{78}$ attribuait les transformations des structures familiales aux changements psycho-affectifs entre parents et enfants, et entre conjoints. Son analyse vaut principalement pour les classes privilégiées de la société. L'heureux rejet des thèses évolutionnistes, le consensus autour de la variabilité des structures et des relations familiales sont plus sensibles dans les synthèses proposées par André Armengaud ${ }^{79}$, François Lebrun $^{80}$, Jean-Louis Flandrin ${ }^{81}$ qui analysèrent systématiquement les nombreuses monographies de démographie, d'histoire sociale que l'abondance soudaine de la recherche avaient accumulées. Le dernier ouvrage cité traduit en anglais fait ouvrage de référence. En Allemagne, Mitterauer et Sieder ${ }^{82}$, à travers une démarche comparative, proposent une vision évolutive plus nuancée de la famille, depuis une situation relativement patriarcale jusqu'au compagnonnage contemporain. Les auteurs, sans parti pris ethnocentriste, analysent la situation des jeunes et des vieux dans la société d'autrefois et dans la société contemporaine. Leur ouvrage contient en outre une bibliographie très complète, s'appuyant sur des textes souvent mal connus portant sur l'Europe de l'Est et du Centre. Il faut citer aussi le très systématique Approaches to the History of the Western Family de Michael Anderson ${ }^{83}$ qui propose une discussion théorique succincte de trois aspects de la famille: l'approche démographique, l'étude des relations affectives, l'aspect économique des relations familiales. Martine Segalen, dans sa Sociologie de la famille $^{84}$, à l'intérieur d'un cadre historique large, propose une réévaluation de la diversité contemporaine des structures et des relations familiales. Les ouvrages de sociologie anglaise qui se présentent souvent sous la forme d'une collection ordonnée d'articles montrent eux aussi leur sensibilité à la dimension historique et le renouvellement de la vision sociologique par l'histoire. Ainsi le remarquable «The Relevance of Family History» de Michael Anderson figure-t-il parmi les plus récentes Sociology of the Family ${ }^{85}$.

Le récent ouvrage de Jack Goody ${ }^{86}$ est d'un autre ordre, car il propose une hypothèse pour expliquer la spécificité du système de

\footnotetext{
78 Lawrence Stone, The Family, Sex, and Marriage in England 1500-1800 (London, 1977). André Armengaud, La famille et l'enfant en France et en Angleterre du XVIe au XVIIIe siècle (Paris, SEDES, 1975), $193 \mathrm{p}$.

${ }_{80}$ François Lebrun, La vie conjugale sous l'Ancien Régime (Paris, Armand Colin, 1975), $179 \mathrm{p}_{81}$

Jean-Louis Flandrin, Familles, parenté, mnison, sexualité dans l'ancienne société (Paris, Hachette, 1976), $287 \mathrm{p}$.

${ }_{82}$ Michael Mitterauer and Reinhard Sieder, The European Family (Oxford, Basil Blackwell, 1982)

${ }_{83}$ Michael Anderson, Approaches to the History of the Western Family (1500-1914) (Londres, MacMillan, 1980).

${ }_{84}$ Martine Segalen, Sociologie de la famille (Paris, Armand Colin, 1981), 283 p.

85 Michael Anderson, Sociology of the Family (1980), 480 p. Première édition 1971.

86 Jack Goody, L'évolution de la famille et du mariage en Europe (Paris, Armand Colin, 1985), $203 \mathrm{p}$
} 
parenté et d'alliance de l'Europe, fondamentalement différent de celui des peuples qui bordaient les rives de la Méditerrannée avant le développement du christianisme. Dans le prolongement de ses comparaisons entre l'Afrique et l'Eurasie, caractérisées, selon lui, par des systèmes différents de dévolution des biens, Goody s'interroge sur les causes historiques de cette différence, et les raisons de la supposée spécificité du système de parenté européen. Celui-ci, on le sait, était caractérisé par des interdits de mariage entre parents proches imposés par l'Église, et des difficultés posées à l'adoption et au remariage. Étudiant le système de parenté des populations de l'Europe avant et aux premiers temps du christianisme, Goody constate que le mariage entre proches parents y était la règle. Pourquoi donc l'Église chrétienne cherchant à conquérir les esprits impose-t-elle des pratiques contraires aux conduites indigènes habituelles? Résumée brièvement, la thèse de Jack Goody est que, par ces restrictions imposées au mariage entre proches, au remariage, à l'adoption, l'Église pouvait détourner vers elle un ensemble de biens et de propriétés qui auraient dû normalement se transmettre au sein des groupes familiaux. L'Église chrétienne limita (consciemment ou non) la nuptialité afin que les biens tombés en déshérance lui soient attribués, ce qui lui permettait d'entretenir des communautés religieuses de personnes qui avaient précisément quitté leur parenté et toute vie active pour une vie monastique.

Au-delà de ces thèses qui auront à être discutées dans les années qui viennent, cet ouvrage nous montre une fois encore la fécondation réciproque entre démarche anthropologique et historique, et aussi un regain d'intérêt théorique pour l'étude de la parenté et de l'alliance dans des sociétés supposées pendant longtemps sans règles.

C'est une des directions vers lesquelles s'orientent à l'heure actuelle un ensemble de travaux d'anthropologues et d'historiens. Divers fichiers sont en cours d'élaboration, car il n'est d'étude possible de l'alliance sans des généalogies assez longues couvrant plusieurs générations, et au minimum sept ou huit. L'anthropologue de la parenté se fait donc par force historien, car ses données se trouvent dans les dépôts d'archives départementales, et non dans la tête de ses informateurs. Auprès de ceux-là, il pourra bien sûr obtenir le vocabulaire de la parenté, le vécu du système. Pourtant, il me semble qu'il faut faire bien attention à ne pas prendre la parole du vieillard aujourd'hui enquêté comme rendant compte d'un passé immémorial. Au contraire, les études historiques nous ont sensibilisés aux transformations qui travaillent sans cesse les populations. Ces recherches individuelles sont menées en liaison avec le développement de programmes informatiques qui vont pouvoir sous peu effectuer des reconstitutions automatiques d'une part, et retrouver des régularités matrimoniales d'autre part. 
Par ailleurs, en France un projet d'ampleur nationale est conduit par des historiens démographes, recherchant un second souffle à travers l'analyse d'un échantillon représentatif de généalogies descendantes. Jacques Dupâquier, le promoteur et l'animateur inlassable de cette grande entreprise écrit qu':

en suivant les individus de la naissance à la mort sans être arrêtés dans leurs reconstitutions par des changements de résidence, les [historiens démographes] pourraient mettre la micro-analyse au service de l'histoire sociale... Les techniques généalogiques doivent permettre deux progrès fondamentaux: étudier des groupes sociaux bien déterminés; analyser la mobilité géographique et sociale au cours des temps. ${ }^{87}$

Une enquête porte sur la France entière à partir d'un échantillon constitué par les descendants de 3000 couples répartis sur tout le territoire national proportionnellement au recensement de 1806. Cet ensemble est formé de toutes les familles portant un patronyme commençant par les lettres TRA.

En Allemagne un autre projet ambitieux est en cours de réalisation sous la direction d'Andrejs Plakans et d'Arthur Imhof; il s'agit du fichier «Schwalm» où, pour grand nombre d'individus, sont reconstitués les généalogies verticales et également le réseau de parenté dont chaque individu pouvait en quelque sorte bénéficier à divers moments de son existence. Le recours à de puissants moyens informatiques et à une large banque de données permet seul ce genre d'analyses.

Il conviendrait cependant de ne pas épuiser toutes ses forces sur la généalogie, objet d'un grand engouement contemporain, tant de la part du grand public que du chercheur. Ce dernier est souvent à la recherche de la variable magique qui devrait permettre de produire des résultats en quelque sorte incontestables parce que quantifiables: le présent bilan montre bien qu'à un moment, ce fut la fiche de famille, à un autre l'étude du groupe domestique, à un autre celui du cycle de la vie familiale. Or il faut savoir articuler méthodes quantitatives et qualitatives, et ne pas s'enfermer dans l'usage d'un unique concept qui risque parfois de devenir un cadre vide qui habille, soit trop large, soit trop étroit, une réalité multiforme et complexe.

De plus, les matériaux sur lesquels s'appuyaient traditionnellement les anthropologues ne sont pas homologues à ceux que l'on peut constituer à partir de sources écrites; l'application de concepts anthropologiques ne doit pas se faire sans une critique rigoureuse. Non seulement ils sont souvent mal adaptés aux sociétés européennes qui ignorent des formations du type clan ou lignage, mais encore ils portent sur

\footnotetext{
87 Jacques Dupâquier, Pour la démographie historique (Paris, Presses universitaires de France, 1984), 160.
} 
des généalogies qui ne sont pas constituées oralement, mais re-constituées à partir de l'état civil ancien. Dans cette perspective, Andrejs Plakians analyse de façon méthodique la possibilité d'adapter les concepts de l'anthropologie de la parenté aux sources documentaires et archivistiques dont l'historien dispose ${ }^{88}$.

On peut clore ce bilan en forme de prospective sur les études relatives à la famille portant sur la ville, bien illustratives du dilemme quantitatif/qualitatif. La ville n'a pas été ignorée de part et d'autre de la Manche, mais les rapprochements scientifiques ont été moins féconds que sur les thèmes précédemment évoqués. Les anthropologues anglais les premiers s'y sont intéressés, qu'il s'agisse de l'ouvrage de Young et Willmott ${ }^{89}$ ou des travaux dirigés par Raymond Firth ${ }^{90}$. Guère d'écho en France, où la ville se trouvait tout entière immergée, mais sans problématique locale dans les études sociologiques des années 1960. Quelques historiens courageux tels Marcel Lachiver sur Meulan ${ }^{91}$ et Jean-Pierre Bardet sur Rouen ${ }^{92}$ ont reconstitué des milliers de fiches de famille; si leur propos central est plutôt démographique, nous apprenons cependant beaucoup sur les questions familiales. Les anthropologues pour leur part semblent plus réticents à s'engager sur ce terrain, bien que l'anthropologie urbaine commence à l'heure actuelle à se constituer comme une branche spécifique de cette discipline en France. Travaillant sur un espace ou un groupe spécifique, le chercheur ne sait comment appréhender la famille dans sa normalité, en dehors du quartier, du groupe ethnique ou social, du fait de la multi-localisation contemporaine des réseaux de parenté.

\section{Conclusion}

Vingt années d'études sur la famille en Europe ont mis en lumière la variété des faits familiaux, et leur complexité. Finies les théories globalisantes comme celle que proposait Talcott Parsons, rejetées les explications mono-causales. Un retournement de perspectives s'est simultanément instauré. La famille n'est plus un objet passif subissant les transformations économiques, sociales, culturelles, mais un lieu de stratégies et de résistances.

Ce bilan montre aussi que l'étude de la famille est désormais intimement mêlée à tous les principaux débats sur les sources historiques,

\footnotetext{
${ }_{88}$ Andrejs Plakans, Kinship in the Past. An Anthropology of European Family Life, 1500 1900 (Oxford, Basil Blackwell, 1984), 276 p.

89 Michael Young and Peter Willmott, Family and Kinship in East London (Londres, Routledge and Nicolson, 1957). 1956)

${ }_{90}$ Raymond Firth, ed., Two Studies of Kinship in London (London, The Athlone Press,

9i Marcel Lachiver, La population de Meulan du XVIIe au XIXe siècle (vers 1600-1870). Étude de démographie historique (Paris, SEVPEN, 1969).

${ }_{92}$ Jean-Pierre Bardet, Rouen aux XVIIe et XVIIIe siècles. Les mutations d' un espace social (Paris, SEDES, 1983).
} 
leurs méthodes, leurs problèmes et interprétations ${ }^{93}$. L'interdisciplinarité si souvent évoquée, si rarement pratiquée, a montré ici sa fécondité. Les ethnologues de la France se font historiens à la recherche d'une documentation qui dépasse les limites de la mémoire de leurs enquêtés, et se donnent les moyens d'analyser les contextes démographiques sociaux et économiques des périodes au long desquelles ils tracent des régularités matrimoniales ou reconstruisent des lignées et des parentèles. De leur côté, les historiens ont lu les anthropologues et réévaluent leurs matériaux avec de nouvelles lunettes conceptuelles recherchant au lieu d'une vague «famille» - terme qui pêche par son imprécision des faits de parenté et d'alliance. En quittant l'étude des Rois et des États pour s'intéresser aux hommes, ils reconstruisent une riche vision du passé quotidien, s'intéressant aux domaines qui étaient généralement ceux de l'ethnologue: les manières de vivre, de se vêtir, de se nourrir ou de penser. La sociologie, pour sa part, a fait reculer les limites chronologiques de son observation, mais souligne à juste titre la nécessité de prendre en compte les catégories sociales et les macromécanismes qui transforment la société. Une Histoire de la famille $e^{94}$ illustre bien cette interdisciplinarité, comme elle met en relief à travers le temps et les civilisations certaines pérennités et la plasticité de l'institution. La prégnance de la famille nucléaire à des époques où on ne l'attendait guère, la capacité des structures traditionnelles de parenté à s'adapter, par exemple en Afrique, aux conditions nouvelles créées par l'industrialisation et l'urbanisation, le détournement au profit de logiques familiales indigènes des règles imposées par le nouvel occupant sont autant de signes du dynamisme familial.

Si ce bilan critique s'est voulu - par convention - limité au champ de la production scientifique européenne, et notablement à celui de la France et de l'Angleterre, il n'a pu éviter de prendre en compte les travaux conduits en Amérique du Nord dont l'influence a été constante. Car des thèmes sont venus de là aussi féconder la recherche en Europe. La démographie historique, la génétique des populations ne seraient pas ce qu'elles sont sans les travaux conduits au Canada; l'étude du concept de cycle de la vie familiale a été remarquablement relancée par Tamara K. Hareven, et c'est d'Amérique du Nord que se développe c'est leur histoire propre qui les y pousse - l'étude de la famille en ville, en milieu industriel, modèles de recherches à suivre pour les anthropologues et historiens à la recherche de sujets d'étude neufs.

L'extraordinaire abondance des recherches sur le vaste thème de la famille continuera-t-elle au même rythme dans les années à venir? Il

\footnotetext{
93 Lawrence Stone, «Family History in the 1980's. Past Achievements and Future Trends», Journal of Interdisciplinary History, 12 (1981): 51-87.

94 André Burguière, Christiane Klapisch, Martine Segalen, Françoise Zonabend, Histoire de la famille (Paris, Armand Colin), 3 vol., sous presse.
} 
ne faut pas sous-estimer les effets de la crise qui privent les centres de recherches du sang nouveau des jeunes chercheurs. Par ailleurs on peut se demander si les incitations de recherche plus centrales sont les plus aptes à stimuler un travail sur un thème dont l'intérêt s'est imposé de la base à partir d'une sorte de crise de conscience collective. Les transformations contemporaines de la famille sont peut-être le meilleur garant d'un prolongement de l'intérêt pour ce sujet inépuisable. 